\title{
Accumulation dynamics of oxygen clusters in silicon and formation of their nonhomogeneous distribution
}

\author{
P.A.Selishchev \\ Kiev T.Shevchenko University, Physics department, 6, Prospekt Glushkova, 02022, Kyiv, Ukraine
}

\begin{abstract}
Kinetics of oxygen distribution variation and oxygen clusters (thermodonors) formation is considered and described by a system of nonlinear equations. Analytic solution is obtained for homogeneous nonstationary distribution of oxygen and its complexes.

It is shown that due to the interaction of oxygen interstitials and their complexes the homogeneous state becomes unstable at some conditions, and spatial periodical distribution develops. Its period lies in the range of 10-1000 ̊.
\end{abstract}

Keywords: silicon, oxygen, complexes, interaction, nonhomogeneous distribution.

Paper received 17.12.99; revised manuscript received 27.12.99; accepted for publication 04.01.00.

\section{Introduction}

Silicon is becoming very important material in the field of electronics and communication. Device production process of silicon is also important and the scale is becoming finer and finer. Electro-phyical properties of silicon appreciably depends on concentration of oxygen and their small clusters. Now it has been found that donor states in heat treated silicon (thermodonors) taking place at $350-500^{\circ} \mathrm{C}$ are small complexes consisted of oxygen atoms [1]. To describe kinetics of oxygen complex formation the differen models were proposed $[2,1]$. Equations for concentrations of complexes containing only one, two, three and four oxygen atoms were analyzed in the framework of these models. It is assumed that the complexes of more than four atoms lose donor properties. They explain many experimental facts. However, there are some difficulties. They are as following. The experimental thermodonor concentration is far more than theoretical one. To form complexes at $350-450^{\circ} \mathrm{C}$ consisting even of two oxygen atoms the diffusion coefficient must be more high than the actual one. And at last, now the nine oxygen complexes having electric activity have been detected.

Theoretical approach developed in present article allows to circumvent these difficulties. First of all, we shall examine equations for complexes containing of arbitrary number of oxygen atoms and obtain analytic solution for homogenous, but nonstationary distribution. Then we shall show the necessary thermodonor concentration can be reached locally. It is possible because at low temperature (less than that of some certain crystals) the homogeneous oxygen distribution (when oxygen is atomically dissolved and incorporated into complexes) seems to be unstable due to nonlinear elastic interaction between oxygen atoms and complexes. Periodical spatial distribution is developed. The nature of the phenomena can be explained qualitatively in the next way. Oxygen interstitials and complexes cause elastic deformation. For some directions they are attracted because the elastic forces are anisotropic in crystal. If the oxygen excess arise in some plane that is normal to this direction, for example owing to fluctuations, then the mobile oxygen would tend to be placed in the same plane. When elastic interaction predominates, the influence of diffusion effects on the homogeneous distribution becomes unstable.

Thus, the oxygen distribution and following thermodonor formation depend on preceding crystal history. Present model substantiates hypothesis $[3,4]$ that oxygen distribution is nonhomogeneous before thermodonor formation.

There are some data about nonhomogeneous distribution of own defects, impurity and their complexes in silicon[4-6]. Manifestation of spatial periodical variation of interstitial and impurity accumulations in irradiated silicon was studied in [8]. Quasiperiodic distribution of boron in boron-implanted silicon was observed in $[9,10]$.

Annealing of silicon crystal results in its non-equilibrium state. Oxygen diffusion increases. (Nonhomogeneity extends if crystal temperature does not exceed the critical 


\section{P.A. Selishchev: Accumulation dynamics of oxygen clusters in silicon...}

one. Otherwise oxygen distribution becomes homogeneous.) Oxygen complexes begin to form in places where oxygen concentration is high.

\section{Physical model and basic equations}

It has been known that single oxygen atoms in silicon crystal are situated in two interstitial configurations separated by energetic barrier $\Delta E$.

One of them is a quasimolecule Si-O-S. Another configuration is metastable. Oxygen interstitials in this configuration do not connect with crystal lattice, and so it is much more mobile.

Taking into account this fact L.I. Murin and V.P. Markevich obtained more high effective diffusion coefficient [4] at low temperature, which can be expressed as

$$
\begin{aligned}
& D=D^{\mathrm{o}} \exp \left(-E_{m} / T\right)+ \\
& +\left(v / v^{*}\right) D^{*} \exp \left(-\left(E_{m}^{*}+\Delta E\right) / T\right)
\end{aligned}
$$

Where $D^{\circ}, E_{m}$ and $D^{*}, E_{m}^{*}$ are the pre-exponentials and migration energies for the first and second configurations. $v$ and $v^{*}$ are frequency factors of probabilities for direct and inverse transformations between these configurations. When crystal temperature is high enough, oxygen atoms dissolved in silicon (their concentration is $n_{1}$ ) become mobile and form immobile complexes that contains two, three,...i,... oxygen atoms (their concentrations are $n_{2}, n_{3}, \ldots, n_{i}, \ldots$, respectively). To describe formation of oxygen thermodonor states, one uses the wellknown set of kinetic equations [1,2]:

$$
\begin{aligned}
& \frac{\partial n_{1}}{\partial t}=-\gamma_{1,2} n_{1}^{2}-\operatorname{div} J \\
& \frac{\partial n_{i}}{\partial t}=-\gamma_{i, i+1} n_{i} n_{1}+\gamma_{i-1, i} n_{i-1} n_{1}
\end{aligned}
$$

$$
i=2, \ldots
$$

Diffusion flux of atomically dissolved oxygen is

$$
J=-D\left(\nabla n_{1}-\frac{n_{1} F}{T}\right)
$$

We take into account elastic force, $F$, that acts on oxygen atoms in the point $r$ from other ones and complexes.

$$
F(r)=-\sum_{i=1} \int \nabla V_{1, i}\left(r-r^{\prime}\right) n_{i}\left(r^{\prime}\right) d r^{\prime}
$$

$V_{1, i}\left(r-r^{\prime}\right)$ are corresponding interaction energies. $\gamma_{i, i+1}$ are directly proportional to diffusion coefficient of oxygen atoms, $D(T) . T$ is a crystal temperature in energy units. We do not interest of boundary phenomena and use the cyclic boundary conditions.

\section{Homogeneous state}

The set (1) - (2) has the only homogeneous solution:

$$
n_{1}(t)=\frac{n_{0}}{n_{0} \gamma_{1,2} t+1}
$$

$$
\begin{gathered}
n_{i}(t)=n_{1}(t)\left(\prod_{l=1}^{i-1}\left(-\gamma_{l, l+1}\right)\right) \times \\
\times \sum_{m=1}^{i} \frac{\left(\frac{n_{1}(t)}{n_{0}}\right)^{\frac{\gamma_{m, m+1}-\gamma_{1,2}}{\gamma_{1,2}}}\left(\gamma_{m, m+1}-\gamma_{j, j+1}\right)}{\substack{j=1 \\
j \neq m}}
\end{gathered}
$$

This solution corresponds to the following initial state: $n_{1}(t=0)=n_{0}, n_{i}(t=0)=0, i \geq 2$. It is assumed that $\gamma_{m, m+1} \neq$ $\gamma_{i, i+1}$ if $m \neq j$.

The oxygen atom concentration monotonically decreases and so for $n_{1}\left\langle n_{0} t>0\right.$. Therefore, if $\left.\left.\gamma_{i, i+1}\right\rangle\right\rangle \gamma_{1,2}$ for each $i>1$ the first item in (7) is much more than others, and one can neglect them. In this case

$n_{i}(t) \approx \frac{\gamma_{1,2}}{\gamma_{i, i+1}} n_{1}(t)$

and

$\frac{n_{i}(t)}{n_{m}(t)} \approx \frac{\gamma_{m, m+1}}{\gamma_{i, i+1}}$.

When dependence of oxygen atom and complex concentrations on time is insignificant, i.e. $\left|\Delta n_{1}\right| / n_{1}=\gamma_{1,2} n_{1}(t) \Delta t\langle<1$ we have quasistationary state during $\Delta t$.

Obtained solution (6)-(7) allows to trace time variation of each complex concentration and ascertain more correctly a correspondence between oxygen complexes and thermodonors comparing experimental and theoretical results.

\section{Instability of homogeneous distribution and formation of spatial periodical one}

To examine the stability of quasi-stationary homogeneous solution we use equations (1) - (2) and consider the behavior of small fluctuations $\delta n_{1}(r, t), \delta n_{i}(r, t)$. Restricting to linear terms in (1), (2) and taking into account $\left.\left.n_{1}(t) \approx n_{0}\right\rangle\right\rangle n_{i}, i>1$ one obtains

$\delta n_{1}(r, t), \delta n_{i}(r, t) \cong \exp (\lambda t+i k r)$

and dispersion equation: 


\section{P.A. Selishchev: Accumulation dynamics of oxygen clusters in silicon...}

$$
\left(\lambda+D\left(\gamma+k^{2}+\alpha k^{2} V(k) / V_{0}\right)\right) \prod_{i=2}\left(\lambda+\gamma_{i, i+1} n_{0}\right)=0
$$

Where

$$
\alpha=n_{0} V_{0} / T, \gamma=2 \gamma_{1,2} / D, V(k)=\int V(r) \exp (-i k r) d r .
$$

The value $\lambda$ depends on crystal and external parameters and at certain ones $\left(\alpha=\alpha_{c}\right)$ Re $\lambda$ changes sign first of all for some certain value and direction of wave-vector $k=k_{c}$. In future wave-vector will hold just that direction. $V(k)$ can be expressed in terms of the Green function for such a crystal. We restrict to the first and the second terms that describe long-range attraction and short-rahge repulsion.

So

$$
V(k)=V_{0}\left(-1+B k^{2}\right),
$$

where $\sqrt{B}$ is of order of several crystal lattice periods. $V_{0}$ is function of the crystal volume change and the moduli of elasticity.

When interaction between oxygen atoms becomes greater than certain threshold value and predominates the influence of diffusion effect, the homogeneous state becomes unstable. Thus, if small positive fluctuations of oxygen concentration arise in some plane, the rest of oxygen atoms would tend to be placed in the same plane. Due to anisotropy of interaction, the zones enriched by oxygen atoms and their complexes form the periodical planes perpendicular to some direction in the crystal, namely $k_{c}$.

The bifurcation value of $a$ obtained from (8) is

$\alpha_{c}=1+2 \sqrt{m_{0} B}\left(\sqrt{m_{0} B}+\sqrt{m_{0} B+1}\right)$ where

Inhomogeneous distribution period at $\alpha \approx \alpha_{c}$ is $2 \pi / k_{c}$,

$$
k_{c}^{2}=\frac{\alpha_{c}-1}{2 \alpha_{c} B}
$$

Numerical evolution shows that the value of $\alpha_{c}$ is slightly more than 1 , and period of heterogeneity changes from 10 to $1000 \AA$ with the parameter variation.

The time of periodical distribution development is determined by oxygen diffusion and depends on crystal tem- perature. Hence the non-uniform state can be observed at some region of temperatures. If crystal temperature is very high the non-uniform state does not develop. But if temperature is very low one develops too long.

\section{Acknowledgements}

The author are grateful to O.A. Leontev for his contributions to technical providing the work.

\section{References}

1. V.M.Babich, N.I.Bletskan, E.F.Venger Oxygen in silicon single crystals. «Interpress LDT», Kyiv, 1997, 240 p.(in Russ.)

2. W.Kaiser, H.L.Frish and H.Reiss Formation of donor state in heat-treated silicon // Phys.Rev.,112,(5), pp.1546-1554(1958)

3. A.N.Kabaldin, V.B.Neimash, V.M.Tsmots, L.I.Shpinar Peculiarities of magnetic susceptibility behaviour of irradiated silicon // Ukrainskiy fizicheskiy zhurnal, 40(10), pp.1079-1082(1995) (in Ukraine)

4. L.I.Murin, V.P.Markevich Creation of thermodonors and mechanism of enhanced oxygen diffusion in silicon// Fizika $i$ Tekhnika Poluprovodnikov, 22(7), pp.1324-1327 (1988) (in Russ.)

5. I.V.Antonova, A.Misiuk, V.P.Popov, S.S.Shaimeev Investigation of oxygen precipitates formation process in silicon // Fizika i Tekhnika Poluprovodnikov, 31(8), pp.998-1002 (1997) (in Russ.)

6. N.T.Bagraev, N.A.Vitovskiy, L.S.Vlasenko, E.V.Mashovets, O.Rakhimov Accumulation of electrically active centers in thermo-treated silicon grown by the Czochralsky method // Fizika $i$ Tekhnika Poluprovodnikov, 17(11), pp.19791984(1983) (in Russ.)

7. P.V.Pavlov, V.I.Pashkov, T.Yu.Chigirinskaia, Lattice of microdefects in silicon // Pis'ma v zhurnal techicheskoy fiziki, 15(4), pp.57-60(1989) (in Russ.)

8. I.I.Kolkovskiy, V.V. Luk'janitsa, Peculiarities of accumulation of radiation defects related to vacancies and interstitials in dislocationless silicon with different oxygen amount // Fizika i Tekhnika Poluprovodnikov, 31,(4), pp.405-409(1997) (in Russ.)

9. M.A.Miasnikov, V.I.Obodnikov, V.G.Seriapin, B.I.Fomin, E.I.Cherepov, Kinetics of impurity redistribution in quasiperiodical structures arising in heavy boron-doped silicon irradiated by boron ions // Fizika i Tekhnika Poluprovodnikov, 31,(3), pp.338-341(1997) (in Russ.)

10. M.G.Stepanov, A.B.Potapov, G.G.Malinetskiy, Formations of dissipative structures in silicon implanted by boron. Preprint № 69, Institute of applied mathematics, Russian academy of sciences, 1997 (in Russ.) 